\title{
White matter hyperintensities are related to pain intensity in an outpatient memory clinic population: preliminary findings
}

This article was published in the following Dove Press journal:

Journal of Pain Research

Tarik T Binnekade', Roberto SGM Perez $^{2, \dagger}$, Andrea B Maier ${ }^{3}$, Hanneke FM Rhodius-Meester ${ }^{4}$, Nienke Legdeur ${ }^{5}$, Marijke C Trappenburg, ${ }^{6,7}$, Didi Rhebergen ${ }^{8-10}$, Frank Lobbezoo $^{11,12}$, Erik JA Scherder ${ }^{1}$

'Department of Clinical Neuropsychology, VU University, Amsterdam, The

Netherlands; ${ }^{2}$ Department of

Anesthesiology, VU University Medical

Center, Amsterdam, The Netherlands;

${ }^{3}$ Department of Medicine and Aged Care, Royal Melbourne Hospital, University of

Melbourne, Melbourne, VIC, Australia;

${ }^{4}$ Department of Human Movement

Sciences, MOVE Research Institute

Amsterdam, Vrije Universiteit Amsterdam,

Amsterdam, The Netherlands; ${ }^{5}$ Department

of Neurology, Alzheimer Center, VU

University Medical Centre, Amsterdam

Neuroscience, Amsterdam, Netherlands;

${ }^{6}$ Department of Internal Medicine, Section

of Gerontology and Geriatrics, VU

University Medical Center, Amsterdam, The

Netherlands; ${ }^{7}$ Department of Internal

Medicine, Amstelland Hospital, Amstelveen,

The Netherlands; ${ }^{8}$ Department of

Psychiatry, GGZ inGeest, Amsterdam, The

Netherlands; ${ }^{9}$ Amsterdam Public Health

Research Institute, Amsterdam, The

Netherlands; ${ }^{10}$ Department of Mental

Health, Amsterdam UMC, Amsterdam, The

Netherlands; "'Department of Orofacial

Pain and Dysfunction, Academic Centre for

Dentistry Amsterdam (ACTA), University of

Amsterdam and Vrije Universiteit

Amsterdam, Amsterdam, The Netherlands;

${ }^{12}$ MOVE Research Institute Amsterdam,

Amsterdam, The Netherlands

${ }^{\dagger}$ Roberto SGM Perez passed away on September 7, 2017

Correspondence: Erik JA Scherder Department of Clinical Neuropsychology, VU University Amsterdam, De Boelelaan 109I, 108I HV, Amsterdam, The

Netherlands

Tel +3 I205988804

Email eja.scherder@vu.nl
Background: The association between pain and dementia is complicated and may depend on underlying brain pathology. It was hypothesized that both medial temporal atrophy (MTA) and global cortical atrophy (GCA) predicted no/mild pain, while white matter hyperintensities (WMH) predicted moderate/severe pain.

Objectives: To study the association between pain intensity and measures of brain pathology, more specifically MTA, GCA, and WMH.

Methods: In total, 115 consecutive patients visiting an outpatient memory clinic were included. In total, diagnoses included dementia $(\mathrm{N}=70)$, mild cognitive impairment $(\mathrm{N}=30)$, and subjective cognitive impairment $(\mathrm{N}=15)$. Without administering stimuli, pain intensity was assessed with the Brief Pain Inventory. MTA, GCA, and WMH were measured with a MRI visual rating scale. Logistic regression analyses to examine the relationship between WMH, MTA, GCA, and self-reported pain intensity (no/mild pain versus moderate/ severe pain) were adjusted for confounders.

Results: Mean age of the patients was 81 years (IQR: 78-85, 53\% female). Moderate/severe pain was reported by $23.5 \%$ and associated with greater $\mathrm{WMH}(\mathrm{OR}=3.34,95 \% \mathrm{CI}=1.01-10.97$, $p=0.047$ ), but not MTA or GCA.

Conclusions: In contrast to the present results, earlier studies have reported either a positive or negative relationship between pain and brain volume. It is suggested that the presence of dementia may explain the absence of a relationship between pain and brain volume. WMH is positively related with pain in an older memory outpatient population. Considering the small sample size, our findings should be interpreted with caution. Hence, our conclusions are preliminary findings, warranting future replication.

Keywords: pain, dementia, Alzheimer's disease, brain atrophy, white matter hyperintensities

\section{Introduction}

To date, most studies on pain in dementia have focused on dementia in general ${ }^{1}$. Earlier studies hypothesized that differences in pain experience between the various subtypes of dementia, and between individuals with and without dementia may be related to differences in neuropathology. ${ }^{2}$ Atrophy in, for example, the hippocampus, anterior cingulate cortex, amygdala, and prefrontal cortex may reduce the affective processing of pain, pain memory, and the anticipatory response to painful stimuli. $^{2}$ However, studies show conflicting results, with both positive ${ }^{3}$ and negative associations between brain atrophy and pain intensity being reported. ${ }^{4,5}$ In addition, white matter hyperintensities (WMH), for example in the spinothalamic 
tract, also referred to as the anterolateral pathway, may lead to an increase in pain experience; this type of pain is paraphrased as deafferentiation pain. ${ }^{2,6}$

A recent study in outpatient memory clinics has shown that older patients with Alzheimer's disease (AD) and mixed dementia (MD; $\mathrm{AD}$ and vascular pathology) are less likely to report pain than older individuals without dementia. $^{7}$ The self-reported pain intensity may also differ between patients with different types of dementia. Patients with $\mathrm{AD}$ report a lower chronic pain intensity than individuals without dementia. ${ }^{8-11}$ Patients with VaD report a higher pain intensity than individuals without dementia. ${ }^{12,13}$ A loss of awareness of pain has been reported by carers of patients with frontotemporal dementia (FTD). ${ }^{14}$ Differences in pain sensitivity between different subtypes of dementia have also been the focus in experimental pain studies. The results of those studies were heterogeneous, reporting, for example, a lower pain sensitivity, ${ }^{15-17}$ no difference, ${ }^{18}$ or even a higher pain sensitivity ${ }^{19}$ in AD compared to individuals without dementia. To date, the only experimental pain study focusing on pain in FTD indicates a strong increase in pain tolerance compared to individuals without dementia. ${ }^{20}$ No studies have been identified that examined pain in patients with dementia with Lewy bodies (DLB). Despite the usefulness of a dementia subtype diagnosis for clinical practice, it may lack specificity for research into the association between neuropathology and pain, given that, even within dementia subtypes, a considerable amount of heterogeneity in neuropathology exists. ${ }^{21,22}$ When studying pain in dementia, it may therefore be more appropriate to group participants on brain atrophy and $\mathrm{WMH}$, rather than on the clinical dementia subtype diagnosis.

The aim of the present study was to examine the association between brain atrophy, $\mathrm{WMH}$, and pain, in a combined group of older individuals both with and without dementia. It was hypothesized that the intensity of pain is negatively associated with brain atrophy and positively associated with WMH.

\section{Material and methods}

\section{Patients}

This cross-sectional study includes patients recruited consecutively at two outpatient memory clinics; the Center of Geriatric Medicine Amsterdam (COGA), VU University Medical Center, Amsterdam, and the Geriatric outpatient clinic (BACO), Amstelland Hospital, Amstelveen, The
Netherlands. Data were collected between April 2014 and November 2015 (COGA), and between May 2015 and November 2015 (BACO). All patients were referred to the outpatient memory clinics with cognitive complaints. Patients were included if they were older than 60 years, had given written informed consent and excluded if they had a primary psychiatric disorder, insufficient command of the Dutch language, or if they indicated verbally and/or non-verbally that they did not wish to participate despite earlier consent. Data were collected conform to the declaration of Helsinki. Approval was obtained from the Ethical Review Board of the VU University Medical Center NL43861.029.13. The cohort consisted of 212 patients with a dementia diagnosis of $\mathrm{AD}, \mathrm{MD}, \mathrm{VaD}$, FTD, DLB, or no dementia referred to as mild cognitive impairment (MCI) or subjective cognitive impairment (SCI). Subsequently, patients were excluded for not completing the pain assessment $(\mathrm{n}=15)$ or not having a magnetic resonance imaging scan (MRI) $(n=82)$, leaving 115 patients available for analysis. The final cohort consisted of $\operatorname{AD}(n=39), \operatorname{MD}(n=20), \operatorname{VaD}(n=8), \operatorname{FTD}(n=1)$, $\operatorname{DLB}(\mathrm{n}=2)$, MCI $(\mathrm{n}=30)$, and SCI $(\mathrm{n}=15)$.

The diagnosis of dementia was based on consensus within a multidisciplinary team, using the National Institute of Neurological and Communicative Disorders and Stroke and the Alzheimer's Disease and Related Disorders Association criteria for dementia due to $\mathrm{AD},{ }^{27}$ the National Institute of Neurological Disorders and Stroke Association Internationale pour la Recherche et l'Enseignement en Neurosciences criteria for vascular dementia, ${ }^{33}$ the revised criteria for FTD ${ }^{31}$ and the revised criteria for $\mathrm{DLB}^{26}$. The diagnosis of dementia included MRI as part of the standard diagnostic process. MCI was diagnosed according to the National Institute on Aging and the Alzheimer's Association criteria. ${ }^{2}$ Finally, the presence of SCI was established when the cognitive complaints could not be confirmed by cognitive testing, and criteria for MCI, dementia, or other neurological or psychiatric disorder known to cause cognitive complaints were not met.

\section{Pain assessment}

The presence and intensity of pain was assessed using the Brief Pain Inventory ${ }^{23}$. Patients were asked to indicate whether they had "pain today other than everyday kinds of pain (eg, toothache, minor headache, or sprains)". Pain intensity was assessed by asking the patients to rate their pain on average and in the last 
$24 \mathrm{hrs}$ on an 11-point (ie, 0-10) numerical scale, with higher numbers indicating more severe pain. Given the high number of patients with no or low pain intensity, this score was dichotomized to represent no/mild pain $(0-3)$ and moderate to severe pain (4-10). ${ }^{24}$

\section{Brain atrophy and $\mathrm{WMH}$}

The presence of brain atrophy and WMH was assessed using MRI visual rating scales. Subjects were scanned on 3.0T MR systems (Titan, Signa, or Discovery MR750). As part of standard clinical care, all scans were visually rated by a trained rater (HR and NL), and subsequently evaluated in a consensus meeting with an experienced neuroradiologist. $^{25}$ All scans included a threedimensional T1-weighted gradient echo sequence and a fast fluid-attenuated inversion recovery (FLAIR) sequence. Brain atrophy was divided into global cortical atrophy (GCA) and medial temporal atrophy. The GCA was assessed using a simplified version of the GCA scale. $^{26,27}$ This visual scale is rated using axial FLAIR images, with scores ranging from 0 to 3 where higher scores indicate more severe atrophy. Medial temporal lobe atrophy (MTA) was assessed using the Scheltens scale $^{28}$ on coronal T1-weighted images. The degree of atrophy was rated using the original criteria by Scheltens and colleagues, based on the height of the hippocampal formation and the width of the choroid fissure and temporal horn, using a 5-point (0-4) scale from the average score of left and right sides. The presence of subcortical WMH was scored on an axial FLAIR, using the Fazekas scale. $^{29}$ The scores on this scale ranged from 0 to 3 , with higher scores indicating more severe WMH. For analyses, the GCA, MTA, and WMH were recoded, because groups with the lowest scores (GCA, MTA, WMH) and highest score (GCA and MTA) were too small to be reliably included in the analysis. Therefore, GCA and WMH were recoded into dichotomous variables, in which (0-1) and (2-3) were grouped. The MTA score was recoded into three groups (0-1), (2), and (3-4).

\section{Mood}

There is ample evidence for the relationship between mood and pain. ${ }^{30,31}$ The short form of the Geriatric Depression Scale (GDS) was used to assess mood. ${ }^{32}$ This 15-item self-report questionnaire has a maximum of 15 points, and has been validated against the ICD-10 and DSM-IV diagnosis of depression in older patients. It has shown acceptable validity and specificity in older patients with a mini-mental state examination (MMSE) score of 15 points and higher. ${ }^{33-35}$ In total, 18 participants had missing values on a maximum of three questions of the GDS. In order to account for this data loss, a relative score was calculated by dividing the number of points on the GDS by the number of questions answered. Three participants had missing values on more than three questions. For these participants, the relative score was coded as missing.

\section{Education}

An adapted version of the Verhage 7-point ordinal rating scale $^{36}$ was used to assess education. The original scale ranges from 1) less than primary education, 2) completed primary education, 3) primary education with incomplete secondary education, 4) complete secondary education, 5) four years of secondary education, and 6) pre-university education and/or higher vocational education, to 7) academic degree. In the adapted version, the first four points have been taken together to represent lower education, while the other points remain unchanged, thus resulting in a 4-point scale.

\section{Medication use}

A list of the prescribed medication provided by the general practitioner or pharmacy was used to rate medication use. The use of analgesic medication (N02 analgesics and M01A NSAID's according to the Anatomical Therapeutic Chemical classification system) was dichotomized into present or absent.

\section{Chronic diseases}

The presence of chronic diseases was rated using the medical chart and information provided by the general practitioner. The chronic diseases were grouped into cardiovascular disease (CVD) and diabetes mellitus. The CVDs include coronary artery diseases, atrial fibrillation, congestive heart failure, cardiomyopathy, transient ischemic attack, cardiovascular accident, and hypertension. The presence of CVD and diabetes was dichotomized into present or absent.

\section{Statistical analyses}

Descriptive data are presented for dementia, MCI, and SCI. Differences in demographic characteristics, pain intensity, medication use, and presence of CVD and diabetes between both groups were assessed using chi-square or Kruskal-Wallis $\mathrm{H}$ tests. The association between pain intensity (0-10) and the presence of pain generating conditions (diabetes, osteoarthrosis, cancerous malignity, 
rheumatoid arthritis, CVD, and depressive symptoms) were assessed using Spearman's rho correlations.

Logistic regression analyses were performed to test the association between GCA, MTA, WMH, and intensity of pain. Multicollinearity was examined by calculating the variance inflation factor (VIF). The VIF of all variables was between 1 and 2, hence multicollinearity was not present. The unadjusted and adjusted associations, including GCA, MTA, and WMH, were computed. All adjusted models included age, gender, education level, relative GDS score, and analgesic use. A $p$-value of $<0.05$ was considered significant. All statistical analyses were performed using IBM SPSS Statistics version 21.0.

\section{Results}

\section{Participant characteristics}

The demographic characteristics are displayed in Table 1. In total, 115 patients were included in the present study, with a median age of 81 years (IQR: $78-85$ years). The association between pain intensity $(0-10)$ and the presence of pain generating conditions (diabetes, osteoarthrosis, cancerous malignancy, rheumatoid arthritis, and cardiovascular disease), as measured with Spearman's Rho analysis, did not reach significance for any of the conditions included. Only depressive symptoms significantly correlated with pain intensity. The dementia, MCI, and SCI groups did not differ significantly with regard to any of the included demographic characteristics, except for the MMSE score, for which the dementia group had a significantly lower score than the MCI and SCI groups $\left(\chi^{2}(2)=28.9, p<0.001\right)$. No difference in $\mathrm{WMH}$ and GCA was found between the dementia, MCI, and SCI groups. The dementia group had significantly higher MTA than the $\operatorname{MCI}\left(\chi^{2}(2)=19.78, p=0.005\right)$ and $\operatorname{SCI}\left(\chi^{2}(2)=31.35\right.$, $p=0.001)$ groups. No difference between MCI and SCI was found with regard to the MTA. In addition, the groups did not differ on any of the included pain characteristics.

\section{Relationship neuropathology and pain intensity}

The results of the logistic regressions are presented in Table 2. There was a significant positive association between WMH and pain intensity. This association was independent of other measures of neuropathology and confounders. In the unadjusted model, a higher amount of WMH was associated with

Table I Demographic characteristics for the dementia, $\mathrm{MCl}$, and $\mathrm{SCl}$ groups

\begin{tabular}{|c|c|c|c|c|}
\hline & \multirow[t]{2}{*}{$\mathbf{N}$} & Dementia & $\mathrm{MCl}$ & SCI \\
\hline & & $(\mathbf{N}=70)$ & $(N=30)$ & $(N=\mid 5)$ \\
\hline Age & 115 & $81.5(77.8-85.0)$ & $81.0(77.8-85.0)$ & $79.5(75.0-83.0)$ \\
\hline Female, n (\%) & 115 & $37(52.9)$ & $15(50.0)$ & $9(60.0)$ \\
\hline Education level (I-4) & 113 & $2.0(I-3)$ & $2.0(2-3)$ & $2.0(2-3)$ \\
\hline MMSE, points $(0-30)$ & 115 & $21.0(17.8-25.0)$ & $26.5(24.0-28.0)$ & $28.0(26.0-30.0)$ \\
\hline GDS $(0-100)$ & 112 & $20.0(6.7-28.8)$ & $20.0(6.7-38.3)$ & $33.3(6.7-53.3)$ \\
\hline CVD present, n (\%) & 112 & $50(71.4)$ & $21(72.4)$ & $7(53.8)$ \\
\hline Diabetes present, n (\%) & 112 & $19(27.1)$ & $7(24.1)$ & $0(0)$ \\
\hline WMH (0-I), n (\%) & 112 & $38(55.1)$ & $13(44.8)$ & $6(42.9)$ \\
\hline GCA $(0-1), n(\%)$ & 113 & $43(62.3)$ & $15(50.0)$ & $7(50.0)$ \\
\hline MTA & 115 & & & \\
\hline MTA 0, n (\%) & & $5(7.1)$ & $10(33.3)$ & $9(60.0)$ \\
\hline MTA I, n (\%) & & $35(50.0)$ & $12(40.0)$ & $2(13.3)$ \\
\hline MTA 2, n (\%) & & $30(42.9)$ & $8(26.7)$ & $4(26.7)$ \\
\hline Moderate-severe pain presence, n (\%) & 110 & $17(25)$ & $6(22.2)$ & $4(26.7)$ \\
\hline Pain intensity, $(0-10)$ & 110 & $0(0-3.50)$ & $0(0-3.00)$ & I $(0-5.00)$ \\
\hline Analgesic use, n (\%) & 115 & $20(28.6)$ & $5(16.7)$ & $6(40.0)$ \\
\hline
\end{tabular}

Notes: Presented as median (IQR) unless specified otherwise. Education level reflects the adapted Verhage scale. GDS is based on a relative score to account for data loss by dividing the number of points by the questions answered. WMH, GCA, and MTA involve recoded scores. Mild-severe pain referrers to an NRS score of 4-I0. GCA is dichotomized based on the scores $\leq I(=0)$ and $\geq 2(=I)$, MTA category 0 included scores $0-I$, category I included score I.5-2, and category 2 included scores $2.5-4$, SCl - subjective cognitive impairment, WMH is dichotomized based on the scores $\leq I(=0)$ and $\geq 2(=I)$. In order to account for this data loss, a relative score was calculated by dividing the number of points on the GDS by the number of questions answered.

Abbreviations: CVD, cardiovascular disease; GCA, global cortical atrophy; GDS, Geriatric Depression Scale; MCI, mild cognitive impairment; MMSE, mini-mental state examination; MTA, medial temporal atrophy; SCl, subjective cognitive impairment; WMH, white matter hyperintensities. 
Table 2 Relationship between GCA, MTA, WMH, and moderate to severe pain intensity

\begin{tabular}{|c|c|c|c|c|c|c|c|}
\hline & & \multicolumn{3}{|c|}{ Unadjusted ( $n=|| 5)$} & \multicolumn{3}{|c|}{ Adjusted $(n=102)$} \\
\hline & & OR & $95 \% \mathrm{Cl}$ & $p$ & OR & $95 \% \mathrm{Cl}$ & $p$ \\
\hline WMH & $\begin{array}{l}0 \\
1\end{array}$ & $\begin{array}{l}\text { I } \\
4.74\end{array}$ & $1.62-13.85$ & $<0.01$ & $\begin{array}{l}1 \\
3.34\end{array}$ & $1.01-10.97$ & 0.047 \\
\hline MTA & $\begin{array}{l}0 \\
1 \\
2\end{array}$ & $\begin{array}{l}I \\
0.82 \\
0.52\end{array}$ & $\begin{array}{l}0.21-3.19 \\
0.12-2.33\end{array}$ & $\begin{array}{l}0.82 \\
0.52\end{array}$ & $\begin{array}{l}\text { I } \\
\text { I.78 } \\
0.88\end{array}$ & $\begin{array}{l}0.31-10.11 \\
0.14-5.58\end{array}$ & $\begin{array}{l}0.52 \\
0.90\end{array}$ \\
\hline $\mathrm{GCA}$ & $\begin{array}{l}0 \\
1\end{array}$ & $\begin{array}{l}\text { I } \\
0.75\end{array}$ & $0.28-1.95$ & 0.55 & $\begin{array}{l}\text { I } \\
0.85\end{array}$ & $0.28-2.63$ & 0.78 \\
\hline
\end{tabular}

Notes: All adjusted models included age, gender, education level, relative GDS score, and analgesic use.

Abbreviations: GDS, Geriatric Depression Scale; GCA, global cortical atrophy; MTA, medial temporal lobe atrophy; WMH, white matter hyperintensity.

a 4.74 times higher risk of reporting a high pain intensity OR $=4.74(95 \%$ CI $=1.62-13.85), p<0.01$. In the adjusted model, a higher amount of WMH was associated with a 3.34 times greater risk of reporting a high pain intensity, $\mathrm{OR}=3.34$ (95\% CI $=1.01-10.97), p=0.047$. No significant association was found between GCA, or MTA, and pain intensity.

\section{Discussion}

This study shows a positive relationship between WMH and self-reported pain intensity in older patients with and without dementia. No relationship was found between GCA, or MTA, and pain intensity. These results were independent of depressive symptoms and other known confounders.

This is the first study to relate the severity of WMH to self-reported pain intensity in patients with dementia and without dementia, limiting comparisons between studies. However, our results are in line with earlier studies of older nursing home patients with "possible" or "probable" vascular dementia who found that nursing home residents with $\mathrm{VaD}$ have a higher self-reported pain intensity than nursing home residents without dementia. ${ }^{12,13}$ Indeed, a similar study reported that older cognitively impaired nursing home patients with a potentially painful condition had a higher chance of reporting pain when they also suffer from diabetes or hypertension, both known risk factors for $\mathrm{WMH}^{37}$ However, our results are not in line with an earlier study of the association between pain intensity and $\mathrm{WMH}$ in older individuals without dementia. ${ }^{38}$ In that study, a relationship between WMH and pain affect, but not pain intensity, was found. Although the severity of WMH has not been reported, the lower age of the participants in that study and the absence of a dementia diagnosis, both factors known to be positively associated with $\mathrm{WMH},{ }^{39,40}$ suggest a lower WMH burden in that population, possibly contributing to the absence of a relationship.

Contrary to our expectation, no relationship between GCA, MTA, and pain intensity was found. The association between brain volume and pain is known to be complicated, given its bidirectional and partly reversible nature. A number of longitudinal studies have found lower brain volume in patients with chronic pain compared to painfree controls. However, after removing the source of the pain, for example hip replacement for hip osteoarthritis, the differences in brain volume between both groups at least partly resolved., ${ }^{4,5}$ Earlier studies reported either a positive or a negative relationship between pain intensity and hippocampal volume in chronic pain patients without dementia. $^{3,41}$ A positive relationship implies the more pain, the larger the volume of the hippocampus. The current study was unable to reproduce these relationships in a sample of older individuals both with and without dementia. The possible increase in hippocampal volume may be explained by the results of the Nun study, and the Baltimore Longitudinal Study of Aging. In these longitudinal studies, it was found that hypertrophy, or increased volume, of the grey matter, in, eg, the hippocampus, progressed from the more anterior regions in nonsymptomatic $\mathrm{AD}$, and $\mathrm{MCI}$ to the more posterior regions, eg, the visual cortex, in AD. ${ }^{42,43}$ Hypertrophy is thought to be an early adaptive mechanism preventing the progression into dementia. ${ }^{44}$ These findings may indicate that the relationship between brain volume and pain is affected by the dementia process in the early stages of dementia. Therefore, brain volume may not be a reliable measure when studying pain in the early stages of dementia. 


\section{Limitations}

Pain intensity was not associated with known pain generating conditions (ie, diabetes, osteoarthritis, cancerous malignity, rheumatoid arthritis, or cardiovascular disease). In addition, the results have not been influenced by a current hip or other fracture given that this would interfere with the measurements of the outpatient memory clinic and none of the participants were suffering from Parkinson's disease. However, a positive correlation between pain intensity and the severity of depressive symptoms was found, which did however not influence the findings of the current study. The lack of a correlation between pain intensity and comorbid conditions may be due to the comorbid conditions not being painful at the time of testing. Variability in the presence of pain is characteristic for conditions like osteoarthritis, where the pain is often not continuously present, but rather waxes and wanes in line with increases in inflammation. In addition, participants were not asked to stop using their pain medication in the week previous to the test day, further limiting the relation between possibly painful conditions and reported pain. Different levels of pain were present in the current study. This is of particular importance, since this paper has been founded on the theory that WMH might provoke pain and might increase the intensity of already present pain. ${ }^{2,6}$ In contrast, grey matter atrophy is thought to reduce pain intensity. ${ }^{4,5}$ Therefore, both participants with moderate-severe pain and no-mild pain are relevant in the current study. In the current study, the clinically relevant pain (ie, moderate-severe pain) is present despite the use of analgesics.

Another limitation of the current study is the lack of specificity of the visual rating scales used to measure MTA, GCA, and WMH, with specifically the GCA and WMH scales lacking information about the way specific brain areas that are involved in the pain matrix are affected. However, although these scales are a subjective interpretation with limited variability, which was further limited in the current study due to methodological considerations. These scales have been used extensively in clinical practice, and provide a good representation of the amount of generalized brain atrophy and WMH relevant for the clinical diagnosis of dementia. Age is an important factor when studying MTA, GCA, and $\mathrm{WMH}^{40}$ and interactions between age and dementia diagnosis have been reported for the MTA and GCA scale. However, for the current study, age is unlikely to have influenced the results, given that our sample was relatively old ( $>75$ years), and the previously reported interactions between age and dementia diagnosis were not present in the population aged $>75$ years. ${ }^{40}$

Power issues frequently hamper dementia research, especially when it involves pain and neuroimaging. In the current paper, only 27 of 115 (23.5\%) of participants reported suffering from moderate-severe pain. A greater number of participants with moderate-severe pain would have allowed us to examine the relationship between pain intensity and underlying pathology in more detail, and simultaneously may have aided in a more robust conclusion on the absence of significant findings for GCA and hippocampal atrophy. However, the prevalence of moderate to severe pain in the current study is in line with the general prevalence of moderate to severe pain in the Netherlands, which has been estimated to be $18 \%{ }^{45}$ In order to compensate for the relatively low number of participants with pain, we chose to perform a logistic regression both unadjusted and adjusted for confounders. This way we were able to report that $\mathrm{WMH}$ are a consistent predictor of pain intensity, since the results remained significant despite the number of predictors varying from 3 (unadjusted) to 8 predictors (adjusted). As has been shown by Vittinghoff \& McCulloch (2007), ${ }^{46}$ in logistic regression the rule of thumb of a minimum of 10 outcome events per predictor variable (EPV) is too conservative, since an EPV of 5-9 produces almost similar results as an EPV of 10-16. However, a lower EPV does increase the chance of missing a positive effect, as is the case for small sample sizes that reduce power. In our study, the combination of these may have resulted in power issues that in turn resulted in the absence of significant findings for GCA and MTA or an overestimate of the effect of WMH. Nevertheless, the hierarchical regression approach of the current study deems this unlikely. Concluding, given the aforementioned limitations the current results should be considered preliminary and warrant further research.

\section{Clinical relevance}

Earlier research has indicated that $\mathrm{WMH}$ are a common feature in both dementia, and aging in general. ${ }^{47,48}$ Given our findings regarding the relationship between $\mathrm{WMH}$ and pain intensity, the high prevalence of $\mathrm{WMH}$ in older patients further highlights the need to focus on white matter changes in order to identify older patients that are at risk of suffering 
from moderate to severe pain. In light of the high prevalence of WMH in the present and earlier studies in older patients both with and without dementia, and their positive relationship with pain intensity, it would be interesting to see whether there is a (central) neuropathic component to the high pain intensity. Further studies should not only further examine the relationship between $\mathrm{WMH}$ and pain, but also focus on clinical predictors of WMH, such as the presence of cardiovascular disease, given that MRI imaging is not always available in dementia care facilities.

\section{Conclusion}

Given the small sample size, our findings should be considered with caution and are of a preliminary nature, requiring replication in larger datasets. $\mathrm{WMH}$, as measured with a MRI visual rating scale, were independently related with a high self-reported pain intensity in a population of older individuals with or without dementia. No relationship was found between GCA, medial temporal atrophy, and pain intensity. Given the high prevalence of $\mathrm{WMH}$ in older individuals both with and without dementia, and their relationship with high pain intensity, more research is needed to clarify whether there is a central neuropathic component to the high pain intensity in these individuals.

\section{Acknowledgments}

We thank the staff of the Centrum voor Ouderengeneeskunde Amsterdam (COGA) and Behandeladvies Centrum Ouderengeneeskunde (BACO) (VU University Medical Center Amsterdam/Amstelland Hospital) for their contribution in collecting the data. We mourn the loss of Professor Doctor Roberto SGM Perez and acknowledge his important contribution to our study of pain in dementia and the conception of the current manuscript. The primary author, Tarik T Binnekade, is funded by Amstelring (RVB U-9107-2013/ $\mathrm{HM} / \mathrm{wb}$ ), Alzheimer Nederland (WE.09-2012-02), Fonds Nuts Ohra (1130-046), Roomsch Catholijk Oude Armen Kantoor (RCOAK) (2010.351), Stichting Beroepsopleiding Huisartsen (SBOH), Stichting Henriëtte Hofje (2010.351H), and the Arnold Oosterbaan Brain Foundation. The findings reported in this article are those of the authors and should not be attributed to any of the funding parties.

\section{Disclosure}

Tarik T Binnekade reports grants from Amstelring, Alzheimer Nederland, Fund Nuts Ohra care subsidies, Roomsch Catholijk Oude Armen Kantoor, Stichting Beroepsopleiding
Huisartsen, Stichting Henriëtte Hofje, and the Arnold Oosterbaan Brain Foundation, during the conduct of the study. F Lobbezoo reports a research grant as a member of Academic Advisory Board from Sunstar Suisse and a research grant from Somnomed outside the submitted work. The other authors report no conflicts of interest in this work.

\section{References}

1. Binnekade T, Van Kooten J, Lobbezoo F, et al. Pain experience in dementia subtypes: a systematic review. Curr Alzheimer Res. 2017;14 (5):471-485. doi:10.2174/1567205013666160602234109

2. Scherder EJA, Sergeant JA, Swaab DF. Pain processing in dementia and its relation to neuropathology. Lancet Neurol. 2003;2 (11):677-686. doi:10.1016/S1474-4422(03)00556-8

3. Barad MJ, Ueno T, Younger J, Chatterjee N, Mackey S. Complex regional pain syndrome is associated with structural abnormalities in pain-related regions of the human brain. J Pain. 2014;15(2):197-203. doi:10.1016/j.jpain.2013.10.011

4. Gwilym SE, Filippini N, Douaud G, Carr AJ, Tracey I. Thalamic atrophy associated with painful osteoarthritis of the hip is reversible after arthroplasty: a longitudinal voxel-based morphometric study. Arthritis Rheum. 2010;62(10):2930-2940. doi:10.1002/art.27585

5. Seminowicz DA, Wideman TH, Naso L, et al. Effective treatment of chronic low back pain in humans reverses abnormal brain anatomy and function. $J$ Neurosci. 2011;31(20):7540-7550. doi:10.1523/ JNEUROSCI.5280-10.2011

6. Wasner G, Lee BB, Engel S, McLachlan E. Residual spinothalamic tract pathways predict development of central pain after spinal cord injury. Brain. 2008;131(Pt 9):2387-2400. doi:10.1093/brain/awn169

7. Binnekade TT, Scherder EJA, Maier AB, et al. Pain in patients with different dementia subtypes, mild cognitive impairment, and subjective cognitive impairment. Pain Med. 2017:1-8. doi:10.1093/pm/pnx162.

8. Scherder E, Bouma A, Slaets J, et al. Repeated pain assessment in Alzheimer's disease. Dement Geriatr Cogn Disord. 2001;12 (6):400-407. doi:10.1159/000051287

9. Scherder E, Bouma A, Borkent M, Rahman O. Alzheimer patients report less pain intensity and pain affect than non-demented elderly. Psychiatry. 1999;62(3):265-272.

10. Scherder EJA, Bouma A. Visual analogue scales for pain assessment in Alzheimer's disease. Gerontology. 2000;46(1):47-53. doi:10.1159/ 000022133

11. Scherder EJA, Eggermont L, Plooij B, et al. Relationship between chronic pain and cognition in cognitively intact older persons and in patients with Alzheimer's disease. The need to control for mood. Gerontology. 2008;54(1):50-58. doi:10.1159/000113216

12. Scherder EJA, Slaets J, Deijen J-B, et al. Pain assessment in patients with possible vascular dementia. Psychiatry Interpers Biol Process. 2003;66(2):133-145. doi:10.1521/psyc.66.2.133.20618

13. Scherder EJA, Plooij B, Achterberg WP, et al. Chronic pain in "probable" vascular dementia: preliminary findings. Pain Med. 2015;16(3):1-29. doi:10.1111/pme.12637

14. Snowden JS, Bathgate D, Varma A, Blackshaw A, Gibbons ZC, Neary D. Distinct behavioural profiles in frontotemporal dementia and semantic dementia. J Neurol Neurosurg Psychiatry. 2001;70 (3):323-332. doi:10.1136/jnnp.70.3.323

15. Benedetti F, Vighetti S, Ricco C, Bergamasco B, Pinessi L, Rainero I. Pain threshold and tolerance in Alzheimer's disease. Pain. 1999;80 (1-2):377-382. doi:10.1016/S0304-3959(98)00228-0

16. Rainero I, Vighetti S, Bergamasco B, Pinessi L, Benedetti F. Autonomic responses and pain perception in Alzheimer's disease. Eur J Pain. 2000;4(3):267-274. doi:10.1053/eujp.2000.0185 
17. Monroe TBT, Gibson SSJ, Bruehl SSP, et al. Contact heat sensitivity and reports of unpleasantness in communicative people with mild to moderate cognitive impairment in Alzheimer's disease: a cross-sectional study. BMC Med. 2016;14(1):74. doi:10.1186/s12916-016-0619-1

18. Jensen-Dahm C, Skau C, Waldemar G. Contact Heat Evoked Potentials (CHEPs) in patients with mild-moderate Alzheimer' s disease and matched control — a pilot study. Pain Med. 2016;17 (4):675-684. doi: $10.1093 / \mathrm{pm} / \mathrm{pnv} 012$

19. Jensen-Dahm C, Werner MU, Dahl JB, et al. Quantitative sensory testing and pain tolerance in patients with mild to moderate Alzheimer disease compared to healthy control subjects. Pain. 2014;155(8):1439-1445. doi:10.1016/j.pain.2013.12.031

20. Carlino E, Benedetti F, Rainero I, et al. Pain perception and tolerance in patients with frontotemporal dementia. Pain. 2010;151 (3):783-789. doi:10.1016/j.pain.2010.09.013

21. Wallin A, Milos V, Sjögren M, Pantoni L, Erkinjuntti T. Classification and subtypes of vascular dementia. Int Psychogeriatr. 2003;15(Suppl 1):27-37. doi:10.1017/S1041610203008937

22. Janocko NJ, Brodersen KA, Ai S-O, et al. Neuropathologically defined subtypes of Alzheimer's disease differ significantly from neurofibrillary tangle-predominant dementia. Acta Neuropathol. 2012;124(5):681-692. doi:10.1007/s00401-012-1044-y

23. Cleeland CS, Ryan KM. Pain assessment: global use of the brief pain inventory. Ann Acad Med Singapore. 1994;23(2):129-138.

24. Woo A, Lechner B, Fu T, et al. Cut points for mild, moderate, and severe pain among cancer and non-cancer patients: a literature review. Ann Palliat Med. 2015;4(4):176-183. doi:10.3978/j. issn.2224-5820.2015.09.04

25. van der Flier WM, Pijnenburg YAL, Prins N, et al. Optimizing patient care and research: the Amsterdam Dementia Cohort. J Alzheimers Dis. 2014;41(1):313-327. doi:10.3233/JAD-132306

26. Pasquier F, Leys D, Weerts JGE, Mounier-Vehier F, Barkhof F, Scheltens P. Inter-and intraobserver reproducibility of cerebral atrophy assessment on MRI scans with hemispheric infarcts. Eur Neurol. 1996;36(5):268-272. doi:10.1159/000117270

27. Harper L, Barkhof F, Fox NC, Schott JM. Using visual rating to diagnose dementia: a critical evaluation of MRI atrophy scales. $J \quad$ Neurol Neurosurg Psychiatry. 2015;86(11):1225-1233. doi:10.1136/jnnp-2014-310090

28. Scheltens P, Leys D, Barkhof F, et al. Atrophy of medial temporal lobes on MRI in "probable" Alzheimer's disease and normal ageing: diagnostic value and neuropsychological correlates. J Neurol Neurosurg Psychiatry. 1992;55(10):967-972. doi:10.1136/jnnp.55.10.967

29. Fazekas F, Chawluk J, Alavi A, Hurtig H, Zimmerman R. MR signal abnormalities at $1.5 \mathrm{~T}$ in Alzheimer's dementia and normal aging. Am J Roentgenol. 1987;149(2):351-356. doi:10.2214/ajr.149.2.351

30. Gerrits MMJG, van Oppen P, Hwj VM, Penninx BWJH, van der Horst HE. Pain and the onset of depressive and anxiety disorders. Pain. 2014;155(1):53-59. doi:10.1016/j.pain.2013.09.005

31. Borsook D. Neurological diseases and pain. Brain. 2012;135(Pt 2):320-344. doi:10.1093/brain/awr271

32. Yesavage JA. Geriatric depression scale. Psychopharmacol Bull. 1988;24(4):709-711.

33. Conradsson M, Rosendahl E, Littbrand H, Gustafson Y, Olofsson B, Lövheim $H$. Usefulness of the geriatric depression scale 15-item version among very old people with and without cognitive impairment. Aging Ment Health. 2013;17(5):638-645. doi:10.1080/ 13607863.2012 .758231
34. Kørner A, Lauritzen L, Abelskov K, et al. The geriatric depression scale and the cornell scale for depression in dementia. A validity study. Nord J Psychiatry. 2006;60(5):360-364. doi:10.1080/ 08039480600937066

35. Lach HW, Chang Y-P ED. Can older adults with dementia accurately report depression using brief forms? J Gerontol Nurs. 2010;36 (5):30-37. doi:10.3928/00989134-20100303-01

36. Verhage F. Intelligentie En Leeftijd: Onderzoek Bij Nederlanders Van Twaalf Tot Zevenenzeventig Jaar. [Intelligence and Age: Investigations on Dutch Persons from Twelve to Seventy-Seven Years]. Assen: Van Gorcum; 1964.

37. Achterberg WP, Scherder E, Pot AM, Ribbe MW. Cardiovascular risk factors in cognitively impaired nursing home patients: a relationship with pain? Eur J Pain. 2007;11(6):707-710. doi:10.1016/j. ejpain.2006.10.006. S1090-3801(06)00171-6 [pii].

38. Oosterman JM, van Harten B, Weinstein HC, Scheltens P, Scherder EJA. Pain intensity and pain affect in relation to white matter changes. Pain. 2006;125(1-2):74-81. doi:10.1016/j. pain.2006.04.030

39. Prins ND, Scheltens P. White matter hyperintensities, cognitive impairment and dementia: an update. Nat Rev Neurol. 2015;11 (3):157-165. doi:10.1038/nrneurol.2015.10

40. Hfm R-M, Benedictus MR, Wattjes MP, et al. MRI visual ratings of brain atrophy and white matter hyperintensities across the spectrum of cognitive decline are differently affected by age and diagnosis. Front Aging Neurosci. 2017;9:1-12. doi:10.3389/fnagi.2017.00117

41. Zimmerman ME, Pan JW, Hetherington HP, Lipton ML, Baigi K, Lipton RB. Hippocampal correlates of pain in healthy elderly adults: a pilot study. Neurology. 2009;73(19):1567-1570. doi:73/19/1567 [pii]10.1212/WNL.0b013e3181c0d454 [doi].

42. Iacono D, O'Brien R, Resnick SM, et al. Neuronal hypertrophy in asymptomatic Alzheimer disease. J Neuropathol Exp Neurol. 2008;67(6):578-589. doi:10.1097/NEN.0b013e3181772794

43. Riudavets MA, Iacono D, Resnick SM, et al. Resistance to Alzheimer's pathology is associated with nuclear hypertrophy in neurons. Neurobiol Aging. 2007;28(10):1484-1492. doi:10.1016/j. neurobiolaging.2007.05.005

44. Iacono D, Markesbery WR, Gross M, et al. The Nun study: clinically silent AD, neuronal hypertrophy, and linguistic skills in early life. Neurology. 2009;73(9):665-673. doi:10.1212/WNL.0b013e3$181 \mathrm{~b} 01077$

45. Breivik H, Collett B, Ventafridda V, Cohen R, Gallacher D. Survey of chronic pain in Europe: prevalence, impact on daily life, and treatment. Eur J Pain. 2006;10(4):287-333. doi:10.1016/j. ejpain.2005.06.009

46. Vittinghoff E, McCulloch CE. Relaxing the rule of ten events per variable in logistic and cox regression. Am J Epidemiol. 2007;165 (6):710-718. doi:10.1093/aje/kwk052

47. Barber R, Scheltens P, Gholkar A, et al. White matter lesions on magnetic resonance imaging in dementia with Lewy bodies, Alzheimer's disease, vascular dementia, and normal aging. J Neurol Neurosurg Psychiatry. 1999;67(1):66-72. doi:10.1136/ jnnp.67.1.66

48. Claus JJ, Staekenborg SS, Roorda JJ, et al. Low prevalence of mixed dementia in a cohort of 2,000 elderly patients in a memory clinic setting. J Alzheimer's Dis. 2016;50(3):797-806. doi:10.3233/JAD-150796 


\section{Publish your work in this journal}

The Journal of Pain Research is an international, peer reviewed, open access, online journal that welcomes laboratory and clinical findings in the fields of pain research and the prevention and management of pain Original research, reviews, symposium reports, hypothesis formation and commentaries are all considered for publication. The manuscript management system is completely online and includes a very quick and fair peer-review system, which is all easy to use. Visit http:// www.dovepress.com/testimonials.php to read real quotes from published authors.

Submit your manuscript here: https://www.dovepress.com/journal-of-pain-research-journal 